\title{
Blank design for deep drawn parts using parametric NURBS surfaces
}

\author{
R. Padmanabhan ${ }^{a, *}$, M.C. Oliveira ${ }^{a}$, A.J. Baptista ${ }^{a}$, J.L. Alves $^{b}$, L.F. Menezes ${ }^{a}$ \\ a CEMUC, Department of Mechanical Engineering, University of Coimbra, Polo II, Pinhal de Marrocos, 3030-201Coimbra, Portugal \\ ${ }^{\mathrm{b}}$ Department of Mechanical Engineering, University of Minho, Campus de Azurém, 4800-058 Guimarães, Portugal
}

\section{A R T I C L E I N F O}

\section{Article history:}

Received 24 July 2007

Received in revised form

19 May 2008

Accepted 23 May 2008

\section{Keywords:}

Blank shape optimization

NURBS surfaces

FEM

DD3IMP

\begin{abstract}
A B S T R A C T
Deep drawing is a forming process controlled by a number of parameters. The initial blank shape is one of the most important process parameter that has a direct impact on the quality of the finished part. This paper describes a new method to determine the optimal blank shape for a formed part using the deformation behaviour predicted by finite element simulations and salient features of NURBS surfaces. The proposed optimization method involves an initial blank shape, which is iteratively trimmed according to the deep drawing simulations results, to achieve the final optimal blank shape. The deep drawing simulations were carried out using DD3IMP, an implicit in-house finite element code. Parametric NURBS surfaces are used to optimize the initial blank shape based on the deformation behaviour predicted by numerical simulation. The proposed method can determine the optimal blank shape for any part within a few iterations.
\end{abstract}

(c) 2008 Elsevier B.V. All rights reserved.

\section{Introduction}

Geometric modelling is extensively used in the design and manufacture of components in aircraft, automobile and shipping industries. It involves the mathematical representation and analysis of surfaces to represent a physical object. Bézier, B-spline, and NURBS are the most commonly used parametric surfaces for geometric modelling. Generalized NURBS surface is preferred over other surfaces in geometric modelling because its evaluation is computationally stable (Sánchez et al., 2003) and its shape can be easily changed through the manipulation of control points, weights or knots as described by Piegl (1991). Dimas and Briassoulis (1999) demonstrated the ability of NURBS to model complex free-form curves and surfaces. In recent years, NURBS surface have been extensively used in CAD applications for product development and manufacturing. Tsai et al. (2003) demonstrated the use of NURBS surface manipulation in CNC machining. A NURBS surface interpolator was used to control tool motion in order to maintain constant feed rate, thus improving the efficiency and quality of the machining. Hu et al. (2001) modified the shape of NURBS surface distributed over multiple control points to improve the aesthetics of products. The use of NURBS curve manipulation can be extended to areas such as sheet metal forming. Sheet metal forming is a complex deformation process controlled by parameters such as the blank shape, tool geometry, sheet thickness, blank holding force, friction, etc. The initial blank shape is one of the important process parameter that has a direct impact on the quality of the finished part, as well as on the final cost of the formed part. Many blank design approaches have been proposed to determine the optimum initial blank shape. Kuwabara and Si (1997) described a slip line field theory based method to determine optimum blank shape. The method is capable of predicting an optimal blank shape within few seconds but assumes the blank material as isotropic, rigid-perfectly plastic and does not deform,

\footnotetext{
* Corresponding author. Tel.: +351 239790700.

E-mail address: padmanabhan@dem.uc.pt (R. Padmanabhan). 0924-0136/\$ - see front matter @ 2008 Elsevier B.V. All rights reserved. doi:10.1016/j.jmatprotec.2008.05.035
} 
i.e., the thickness of the blank does not change during the deep drawing operation. Guo et al. (2000) described finite element method based inverse approach to determine optimum blank contour for industrial parts. The approach uses the knowledge of discretized 3D shape of the final part. The efficiency and convergence rate is dependant on the assumed initial blank. Kim et al. (2000) proposed a roll-back method to predict optimum blank shapes for industrial parts. The deformed blank shape is compared with the target shape and necessary modification is carried out in the initial blank. Park et al. (1999) obtained optimal blank shape for a part using a deformation path iteration method. In all these approaches, either the finite element mesh size was altered during the optimization procedure or only the part's flange area was considered. The focus of this paper is to develop a method to determine the optimal blank shape for a deep drawn part using finite element method and NURBS surfaces. A new algorithm based on the deformation history of the blank and utilizing the salient features of NURBS surfaces is developed and tested to prove that it can be an economical solution in reducing time and material. A rectangular cup is considered for research purposes and numerical tools such as DD3IMP (Menezes and Teodosiu, 2000), DD3TRIM (Baptista et al., 2006) were used to determine the optimal blank shape. In the following section, the numerical tools used and the proposed blank shape optimization procedure are described. In Section 3, results from the simulations are presented, followed by conclusions in Section 4.

\section{Numerical tools used in blank shape optimization procedure}

\subsection{NURBS surface}

A NURBS surface is the rational generalization of the tensorproduct non-rational B-spline surface (Piegl, 1991) and is defined as:

$\mathbf{S}(u, v)=\frac{\sum_{i=0}^{n} \sum_{j=0}^{m} w_{i, j} \mathbf{P}_{i, j} N_{i, p}(u) N_{j, q}(v)}{\sum_{i=0}^{n} \sum_{j=0}^{m} w_{i, j} N_{i, p}(u) N_{j, q}(v)}$

where $w_{i, j}$ are the weights, $\mathbf{P}_{i, j}$ form a control net, and $N_{i, p}(u)$ and $N_{j, q}(v)$ are the normalised B-splines of degree $p$ and $q$ in the $u$ and $v$ directions, respectively. The ith $B$-spline basis function of $p$-degree (order $p+1)$, denoted by $N_{i, p}(u)$, is defined as

$$
\begin{aligned}
& N_{i, 0}(u)= \begin{cases}1 & \text { if } u_{i} \leq u<u_{i+1} \\
0 & \text { otherwise }\end{cases} \\
& N_{i, p}(u)=\frac{u-u_{i}}{u_{i+p}-u_{i}} N_{i, p-1}(u)+\frac{u_{i+p+1}-u}{u_{i+p+1}-u_{i+1}} N_{i+1, p-1}(u)
\end{aligned}
$$

defined on the knot vectors

$$
\begin{aligned}
& \mathrm{U}=\left[0,0, \ldots, 0, u_{p+1}, \ldots, u_{n}, 1,1, \ldots, 1\right] \\
& \mathrm{V}=\left[0,0, \ldots, 0, v_{q+1}, \ldots, v_{m}, 1,1, \ldots, 1\right]
\end{aligned}
$$

where the end knots are repeated with multiplicities $p+1$ and $q+1$, respectively, in order to guarantee that the surface is at least $C^{1}$ continuous at the end knots (Piegl and Wayne, 1997).

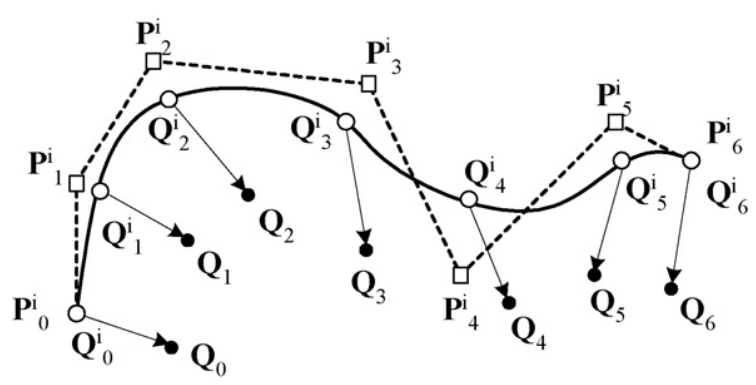

Fig. 1 - Example on defining the final and new initial set of points.

The shape of the NURBS surface can be modified either by moving control points, by changing knot vectors or by changing the weights. These strategies are used to change the surface locally, were needed. In the present optimization procedure, all interpolation points may change their position; hence, the NURBS surface is modified through a simple push/pull technique applied to the set of points interpolated by the surface. The push/pull technique is directly applied to generate NURBS curve. This curve defines the new blank shape and it is extruded to a NURBS surface only to allow its use as trimming domains for a solid finite element mesh.

Suppose an initial curve $C^{i}(u)$, like the one presented in Fig. 1 , is defined by $n+1$ control points $\mathrm{P}^{\mathrm{i}}$. A set of $n+1$ points $\mathrm{Q}^{\mathrm{i}}$ on the $\mathrm{C}^{\mathrm{i}}$ curve is defined, based on the minimum distance to each control point.

The simple push/pull technique is applied to each of the $Q^{i}$ points to determine their new positions, defined as $Q$, as exemplified in Fig. 1. The new curve must interpolate the set of $n+1$ points $Q$. Given this set of points, to interpolate them with a pth-degree non-rational B-spline curve, it is necessary to select the weights and an appropriate knot vector. Very little has been published about the choice on weights for the fit in process. As demonstrated by Piegl and Wayne (1997), for interpolation, there is probably no reason for choosing weights different from 1, in particular when the number of control points is defined. There are many methods for choosing the knots, most of them heuristic. In this paper, the chord length method is selected, which is the most widely used since the method provides an approximated uniform parameterization of the curve. This method assigns a parameter value $\bar{u}_{k}$ to each point of the set $\mathbf{Q}_{k}, k=0, \ldots, n$, based on the distance between them

$d=\sum_{k=1}^{n}\left|\mathbf{Q}_{k}-\mathbf{Q}_{k-1}\right|$,

$\bar{u}_{0}=0, \quad \bar{u}_{n}=1 \quad$ and $\quad \bar{u}_{k}=\bar{u}_{k-1}+\frac{\left|Q_{k}-Q_{k-1}\right|}{d} \quad k=1, \ldots, n-1$.

The knot vector is defined following the technique of averaging

$$
\begin{aligned}
& u_{0}=\cdots=u_{p}=0, \quad u_{r-p}=\cdots=u_{r}=1 \\
& \text { and } \quad \bar{u}_{j+p}=\frac{1}{p} \sum_{i=j}^{j+p-1} \bar{u}_{i}, \quad j=1, \ldots, n-p,
\end{aligned}
$$


where $r=n+p+1$. This technique guarantees that the knots reflect the distribution of $\bar{u}_{k}$. Once the weight and knot vectors are defined, to determine the $p$ th-degree non-rational B-spline curve $C$, it is necessary to set up the $(n+1) \times(n+1)$ system of linear equations

$\mathbf{Q}_{k}=C\left(\bar{u}_{k}\right)=\sum_{i=0}^{n} N_{i, p}\left(\bar{u}_{k}\right) \mathbf{P}_{i}$,

where the control points, $\mathbf{P}_{i}$, are the $n+1$ unknowns. In threedimensional space, the system, Eq. (5), has three right-hand and left-hand sides, each corresponding to one of the directions, that allow to determine coordinates of the control points.

The selection of the initial set of points to which the pull/push technique will be applied is based on the minimum distance between the control points of the initial curve $C^{i}$, designated by $\mathrm{P}^{\mathrm{i}}$, and the nodes of the initial mesh closest to these points. The selected nodes in the initial mesh, designated by $\mathrm{Q}^{\mathrm{i}}=\mathrm{X}^{\mathrm{init}}$, change their position due to the draw-in during deep drawing process. The intersection of their trajectory and a required target contour defines an intersection position named $\mathrm{X}^{\text {inter }}$. The knowledge of the initial position $\mathrm{X}^{\text {init }}$, the final position $\mathrm{X}^{\text {final }}$, and the intersection position $\mathrm{X}^{\text {inter }}$ enables the push/pull technique to calculate the positions of the new set of control points,

$\mathrm{Q}_{k}=\mathrm{X}_{k}^{\mathrm{init}}+\left(\mathrm{X}_{k}^{\text {inter }}-\mathrm{X}_{\mathrm{k}}^{\mathrm{final}}\right)$.

The vector $\mathbf{X}^{\text {inter }}-\mathrm{X}^{\text {final }}$ defines the direction and the distance to move each of the initial nodes selected.

Application of this push/pull technique is exemplified in Fig. 2. For point $j$, since its final position is located inside the target contour, the point in the new NURBS surface has to move outside the original NURBS surface. On contrary, since the final position of point $j+1$ is located outside the target contour, the point in the new NURBS surface has to move inside the original NURBS surface. This push/pull technique can also be applied using a damping factor $\xi$, to smooth the oscillations that may occur between the several NURBS surfaces produced during the iterative procedure. This corresponds to changing Eq. (8) to

$\mathbf{Q}_{k}=\mathbf{X}_{k}^{\text {init }}+\xi\left(\mathbf{X}_{k}^{\text {inter }}-\mathbf{X}_{k}^{\text {final }}\right)$

\subsection{DD3IMP}

Drawing simulations were carried out using the in-house finite element code DD3IMP (contraction of Deep Drawing 3d Implicit code), developed by Menezes and Teodosiu (2000). DD3IMP is developed specifically to simulate sheet metal forming processes. The evolution of the deformation process is described by an updated Lagrangian scheme. An explicit approach is used to calculate an approximate first solution for the nodal displacements, the stress state and frictional contact forces. This first trial solution of the configuration of the deformable body is therefore iteratively corrected. This correction phase is done implicitly using a Newton-Raphson algorithm and finishes when a satisfactory

\section{Table 1 - Global algorithm of DD3TRIM}

Pre-processing stage

Read initial mesh and trimming NURBS surface

Classify elements as keep, eliminate, treat based on their location in relation to the NURBS surface

Correction stage

For all the elements with treat status calculate the volume in the zone to be eliminated $V^{\text {eliminate }}$

IF V $V^{\text {eliminate }}>50 \% \Rightarrow$ eliminate the element

IF Veliminate $\leq 50 \% \Rightarrow$ treat the element

Project the affected nodes towards the trim surface

Post-processing stage

Degenerate or distribute nodes in the element with pentahedral shapes

Update nodal coordinates and connectivity tables

equilibrium of the deformable body is achieved. It is then possible to update the blank sheet configuration at the end of time increment, as well as all the state variables, passing on to the calculation of the next increment until the end of the process. The Coulomb's classical law models the friction contact problem between the rigid bodies (tools) and the deformable body (blank). The contact with friction problem is treated by an augmented Lagrangian approach. The abovementioned fully implicit Newton-Raphson scheme is used to solve, in a single loop, all the non-linearities associated to the problem of contact with friction and the elastoplastic behaviour of the deformable body as described by Oliveira et al. (2007).

\subsection{DD3TRIM}

In deep drawing processes, the average element size influences results like draw-in prediction, depending upon the complexity of the final shape of the part. In blank shape optimization, it is important to fix this numerical parameter and avoid meshing procedures. In the proposed method, a base mesh is always trimmed to define the initial and intermediate blank shapes, in accordance with the initial NURBS surface or those created by the algorithm. This eliminates the influence of the finite element size in the deformation of the blank. The trimming operation is performed with DD3TRIM, a numerical tool developed by Baptista et al. (2006) to trim solid finite element meshes, based on the trimming domains defined by a NURBS surface. The global algorithm of the trimming tool is described in Table 1.

The surface normal orientation defines the redundant elements to be eliminated. Each finite element is classified between "keep", "eliminate", or "treat", based on the analysis of its constituent nodes geometrical position relative to the NURBS surface. The elements that are classified as to "treat" are processed by evaluating the volume of the element in the zone to be removed.

\section{Blank shape optimization procedure}

Numerical analysis including finite element methods can simulate sheet metal forming processes and provide useful information, thus reducing experimental trial and error cycles. Numerical models are simple to analyse and easy to modify, 


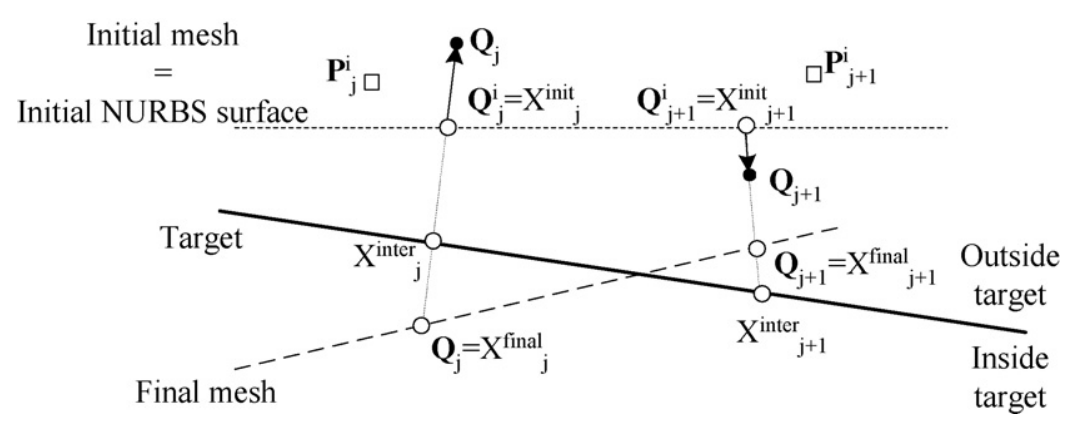

Fig. 2 - Example on the application of the pull/push technique.

which results in the elimination of wastage and in the manufacture of better and cheaper components. However, prior knowledge on the mechanical behaviour of the blank sheet will simplify the process of identifying optimal process parameters. Given the geometry of the drawn part, the mechanical properties of the blank and the friction conditions, the blank holder force and punch forces and the initial blank geometry can be determined through intuition or using empirical formulae. Nonetheless, a systematic approach in establishing these process parameters is necessary to reduce wastage in material and time. Especially, when it is possible to produce near net-shape parts, the wastage from removing excess material at the flange should be eliminated.

Fig. 3 illustrates the proposed blank shape optimization procedure. The initial process parameters like, the tools geometry, the mechanical properties of the blank sheet, the friction conditions and the blank holder force are fixed during the optimization procedure. Generally, to accommodate the continuous variation of the nodal coordinates in optimization, a time-consuming remeshing procedure is employed. In the proposed method, a regular and uniform mesh with dimension large enough to accommodate the probable blank shapes is defined as the base mesh for trimming, as described in the previous section. The initial blank shape can be determined with the aid of empirical formulae. Based on this information, a corresponding NURBS surface is produced, and the base mesh is cut to generate the finite element mesh. The number of control points of this initial NURBS surface defines the number of design variables. The same number of control points will be used in the determination of the new NURBS surfaces during the optimization procedure. At the end of every deep drawing simulation, the closest nodes to the control points of the NURBS surface are identified. Each nodes initial, $\mathrm{X}^{\text {init }}$, and final, $\mathrm{X}^{\mathrm{final}}$, positions define a straight line which approximates the flow path. This straight line intersects the target contour at a point, $\mathrm{X}^{\text {inter }}$ as illustrated in Fig. 2. The new set of points to be interpolated is computed using Eq. (9). The NURBS surface that interpolates this set of points is determined as described in Section 2.1. The global algorithm of the correction stage of the optimization procedure is summarized in Table 2.

The user-defined damping coefficient $\xi$ introduced in the push/pull technique is applied to arrest flange contour oscillation between extremum. Once the new coordinates of all control points are determined, the corresponding NURBS curve is extruded to produce an intermediate NURBS surface, which is used to generate an intermediate mesh from the base

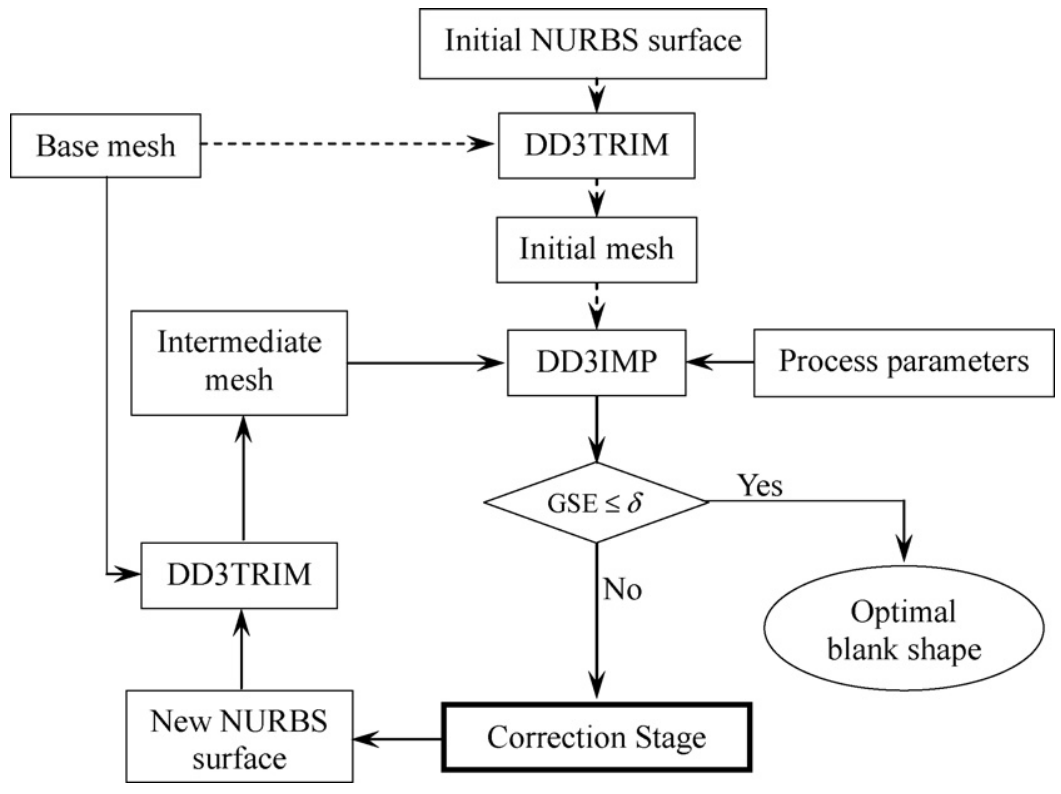

Fig. 3 - Blank shape optimization procedure. 


\section{Table 2 - Global algorithm of correction stage}

Pre-processing stage

Read final configuration of the mesh from previous simulation

Read initial/intermediate NURBS surface

Select the set of points $\mathrm{Q}^{\mathrm{i}}=\mathrm{X}^{\text {init }}$ based on the minimum distance to the control points, $P_{i}$ of the initial/intermediate NURBS curve Determine the straight line defined by the two points $\mathrm{X}^{\text {init }}$ and $\mathrm{X}^{\text {final }}$

Determine the intersection between the straight line and the target contour to define $\mathrm{X}^{\text {inter }}$

Calculate GSE error

If GSE $\leq \delta$, exit procedure since the algorithm has achieved optimal initial blank for the given part

Else, apply push/pull technique

Define the damping coefficient $\xi$

Calculate the new set of points to be interpolated $\mathrm{Q}=\mathrm{X}^{\mathrm{init}}+\xi\left(\mathrm{X}^{\text {inter }}-\mathrm{X}^{\mathrm{final}}\right)$

Calculate new intermediate NURBS curve

Extrude the NURBS curve to define intermediate NURBS surface

mesh using DD3TRIM. Starting from this intermediate mesh, the new deep drawing simulation is performed with DD3IMP. The procedure is repeated until an optimal blank shape that results in a flange contour with negligible deviation from the target contour is obtained. In order to quantify the deviation between the flange and the target contours, a geometrical measure namely, geometrical shape error, is used. Geometrical shape error (GSE), expressed in $\mathrm{mm}$, is defined as the root mean square of the shape difference between the target shape and the deformed shape as in the following equation (Park et al., 1999):

$\mathrm{GSE}=\sqrt{\frac{1}{n} \sum_{i=1}^{n}\left|\mathrm{X}^{\text {inter }}-\mathrm{X}^{\mathrm{final}}\right|^{2}}$

The distance between $\mathrm{X}^{\text {init }}$ and $\mathrm{X}^{\mathrm{final}}$ is evaluated at the end of each simulation, and $n$ is the number of control points used in the initial NURBS surface. When the GSE reaches a value less than " $\delta$ ", a value predetermined by the user for a required accuracy in the flange shape, the iterative procedure is stopped because the optimal blank shape for the part has already been obtained.

\section{Application to a rectangular cup and cross tool examples}

Rectangular cup geometry is used in this study to demonstrate the robustness of the proposed methodology in determining the optimal blank shape. In addition, a cross tool geometry is used to show the versatility of the proposed method. The examples are different and have complex flow characteristics; hence, chosen for this study on optimal blank shape determination. The cross tool forming produces most of the industrial strain paths such as, simple tension, plane strain, shear and biaxial stretching. Cross tool geometry is used by Renault to benchmark new materials as described by Maeder (2005). The aim of using these examples is to show that the proposed method can easily be applied to any formed part in the determination of optimal initial blank shape.
Table 3 - Material properties of mild steel (DC06)

\begin{tabular}{lc}
$E(\mathrm{GPa})$ & 210.0 \\
$\mathrm{Y}_{0}(\mathrm{MPa})$ & 123.6 \\
$\mathrm{~K}(\mathrm{MPa})$ & 529.5 \\
$n$ & 0.268 \\
$v$ & 0.3 \\
$r_{0}$ & 2.53 \\
$r_{45}$ & 1.84 \\
$r_{90}$ & 2.72 \\
\hline
\end{tabular}

Mild steel (DC06) blank of $0.8 \mathrm{~mm}$ thickness is used for both examples in this study and the material properties are listed in Table 3, where $E$ is the Young's modulus, $Y_{0}$ the yield stress, $v$ the Poisson's ratio, $n$ and $K$ are the material properties according to the Swift law which describes isotropic work-hardening equation:

$\sigma=K\left(\varepsilon_{0}+\bar{\varepsilon}^{\mathrm{P}}\right)^{n}$

where $\sigma$ is the equivalent stress, $\bar{\varepsilon}^{\mathrm{P}}$ the equivalent plastic strain and $\varepsilon_{0}=\left(\mathrm{Y}_{0} / \mathrm{K}\right)^{1 / n}$.

The material follows Hooke's law in the elastic domain; orthotropic behaviour is described by the classical Hillǐ48 quadratic yield criterion, proposed by Hill (1948). Initial process parameters were chosen based on empirical relations and optimal values. Blank holder forces of $800 \mathrm{~N}$ and $72.5 \mathrm{kN}$ and friction coefficients of 0.08 and 0.03 were used for rectangular cup and cross tool geometries, respectively. The process parameters remained the same through the optimization procedure. The forming tools geometries used in this study are presented in Fig. 4(a) for rectangular cup and Fig. 4(b) for cross tool.

Numerical simulations were carried out for only one quarter of the geometries due to symmetry of the part. For the rectangular cup, the initial blank shape is determined based on the empirical formulae proposed by Barata da Rocha and Ferreira Duarte (1993), and a corresponding NURBS surface is produced. A base mesh is produced with an average element size of $1.4 \mathrm{~mm}$. This base mesh is cut with initial NURBS surface to produce the initial finite element mesh. The initial flange contour corresponding to the blank shape obtained using empirical formulae is presented in Fig. 5, as well as the flange contour at the end of the punch stroke. In this particular case of the rectangular cup, three lines (1-3) define the target contour, also indicated in Fig. 5. Straight line equations $(y=30$; for $x<26$ and $x=45$; for $y<11)$ for lines 1 and 3 , respectively, and the circle equation $\left[(x-26)^{2}+(y-11)^{2}=(19)^{2}\right]$ are used in the algorithm to define the target contour. Only the first quadrant of the circle equation is considered for computing deviations across line 2. For the cross tool, the initial blank shape is a square with $125 \mathrm{~mm} \times 125 \mathrm{~mm}$ dimensions. The required flange contour for the cross tool is $100 \mathrm{~mm} \times 100 \mathrm{~mm}$ with an arc tangent of $50 \mathrm{~mm}$ radius at the corner. A draw depth of $60 \mathrm{~mm}$ is used for the cross tool. The flange contours considered in both cases are defined by straight lines and curves. In industrial applications, the flange contour may not constitute simple geometries, in which case the algorithm takes a NURBS curve defining the required target contour as the reference. The algorithm presented in this paper assumes 

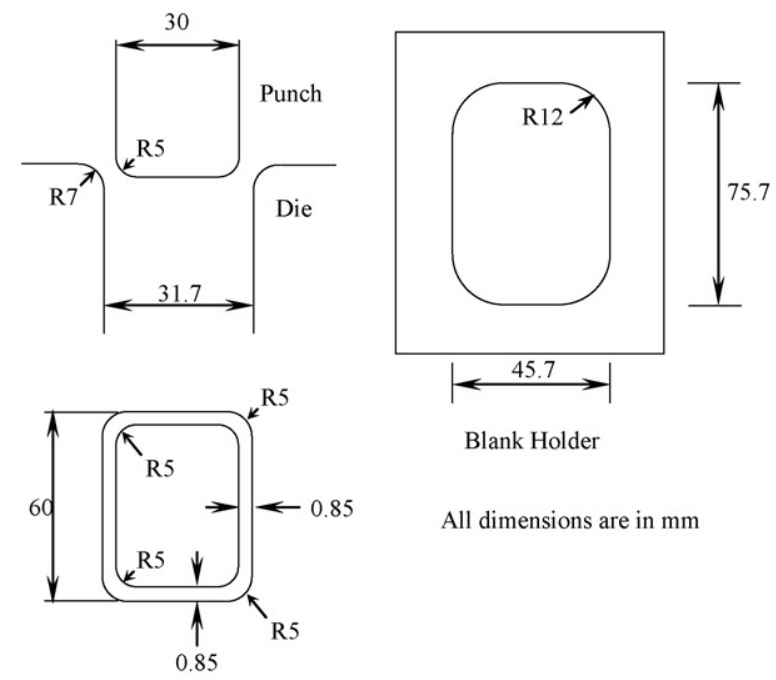

Blank Holder

All dimensions are in $\mathrm{mm}$

(a)
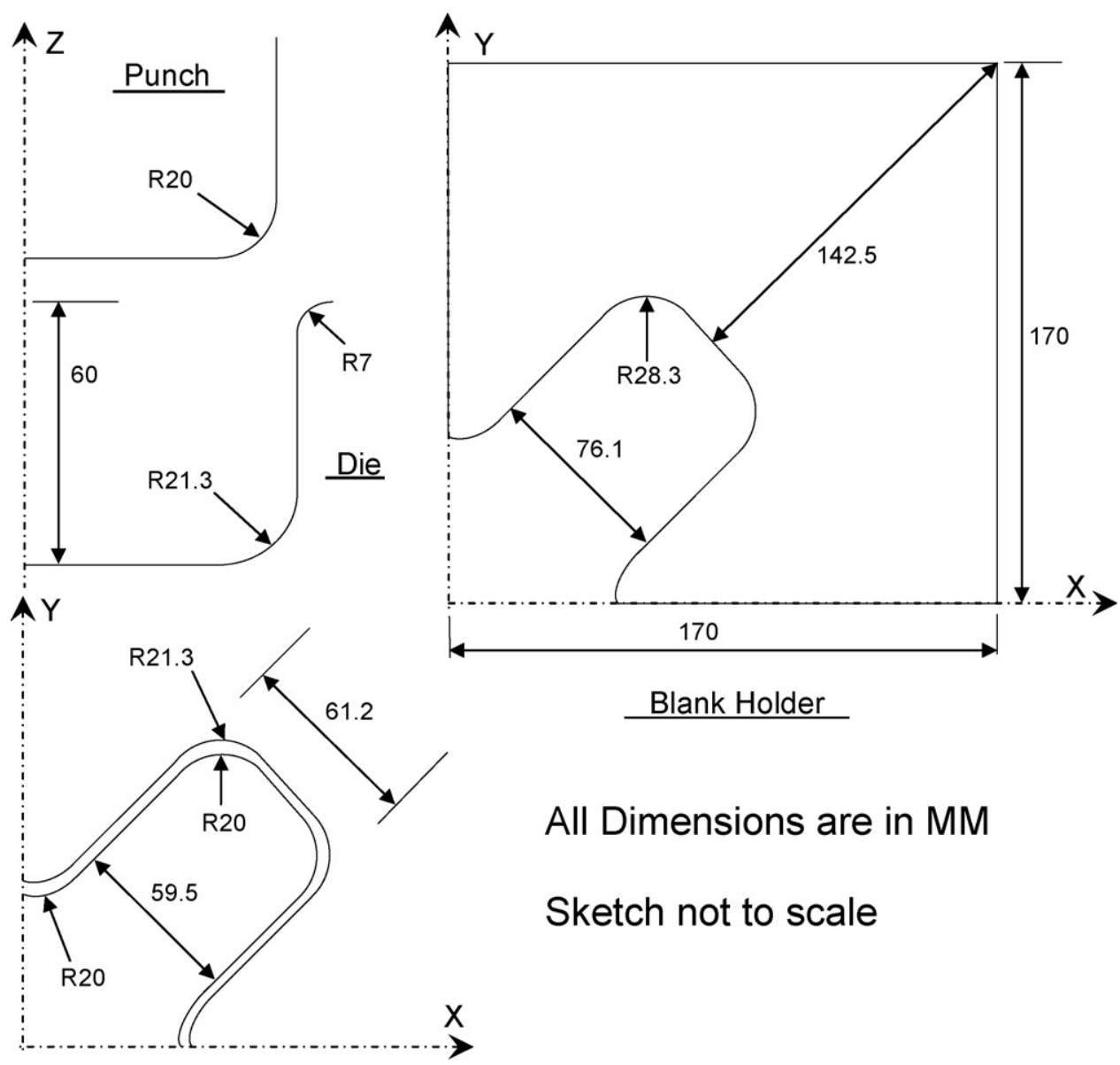

Blank Holder

\section{All Dimensions are in MM}

Sketch not to scale

(b)

Fig. 4 - (a) Geometry of the rectangular cup forming tools and (b) geometry of one quarter of the cross forming tools.

that the flow path, for both geometries, resemble a straight line.

Fig. 5 shows the trajectory of nodes closest to the control points as the deep drawing process progress in the first simulation of the rectangular cup example. The positions of nodes are drawn at intervals of $5 \mathrm{~mm}$ starting from $10 \mathrm{~mm}$ draw depth until a final depth of $30 \mathrm{~mm}$. The trajectories of the nodes remain almost a straight line during deep drawing. Hence, confirming the option of assuming in the correction stage, that the movement of each node follows a straight line. 


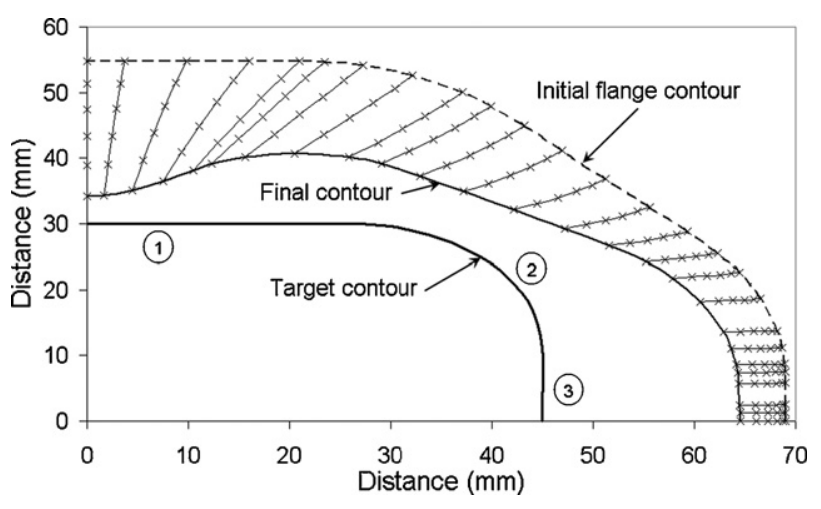

Fig. 5 - Trajectory of nodal points during deformation.

\section{Table 4 - Control points and damping coefficients}

Damping coefficient

Control points
0.6

16
0.8

11
A preliminary sensitivity study was carried out to determine the influence of the damping coefficients and the number of control points in the NURBS curve on the shape error. The results presented are only for the rectangular cup geometry.

\subsection{Influence of damping coefficient}

The influence of damping coefficient on achieving the optimum solution is studied in this section. Four different values, listed in Table 4, were considered.

The GSE allows correct estimation of the distance between the actual flange contour and the target contour. However, by definition it is not possible with the GSE to evaluate whether the actual flange contour is more inside or outside the target contour. To clearly understand the shape error, a measure called target shape error (TSE) is used to quantify the deviation of the flange contour from the required target contour. Target shape error, expressed in $\mathrm{mm}$, is defined by the following equation:

$\mathrm{TSE}=\frac{1}{n} \sum_{1}^{n} f\left(\frac{\left|\mathrm{X}^{\text {init }}-\mathrm{X}^{\mathrm{final}}\right|}{\left|\mathrm{X}^{\text {init }}-\mathrm{X}^{\text {inter }}\right|}\right)\left|\mathrm{X}^{\text {inter }}-\mathrm{X}^{\mathrm{final}}\right|$

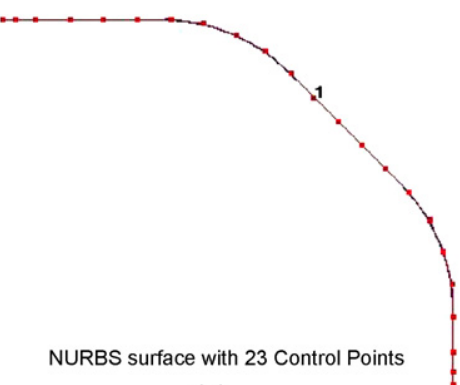

(a)

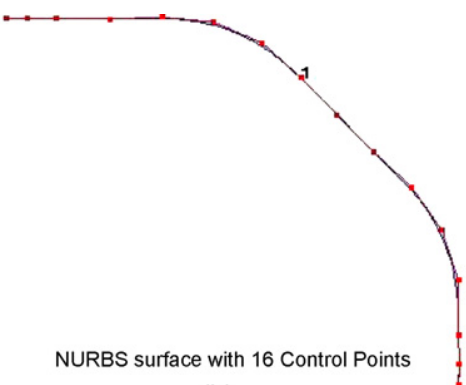

(b)

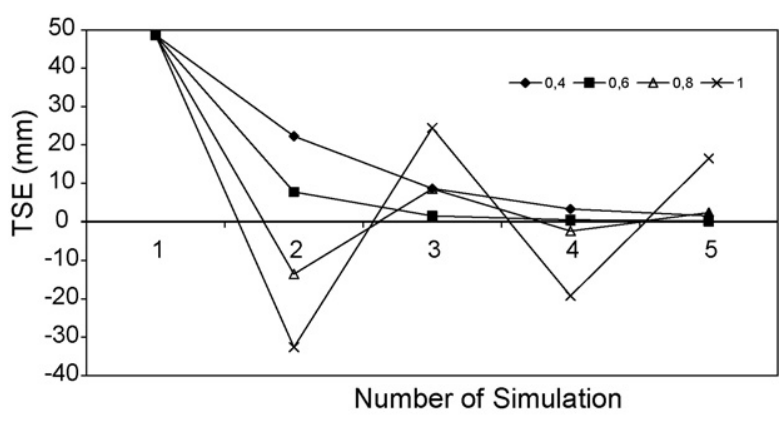

Fig. 6 - Influence of damping coefficient on TSE. where

$f\left(\frac{\left|X^{\text {init }}-X^{\text {final }}\right|}{\left|X^{\text {init }}-X^{\text {inter }}\right|}\right)=\left\{\begin{array}{cc}1 & \text { if } \frac{\left|X^{\text {init }}-X^{\text {final }}\right|}{\left|X^{\text {init }}-X^{\text {inter }}\right|}<1 \Rightarrow \text { outside target } \\ -1 & \text { if } \frac{\left|X^{\text {init }}-X^{\text {final }}\right|}{\left|X^{\text {init }}-X^{\text {inter }}\right|}>1 \Rightarrow \text { inside target }\end{array}\right.$

The TSE allows correct estimation of the distance between the actual flange contour and the target contour and to determine the total deviation, shown in Fig. 6. Each damping coefficient scheme started with the result from the first iteration and hence with a starting TSE of $48.6 \mathrm{~mm}$, which corresponds to a contour completely outside the target contour.

Large target shape error observed in the first iteration and the flange contour oscillation thereafter (when $\xi=0.8$ and 1.0) is due to the large initial blank shape obtained from the empirical equations. Due to the large initial blank shape, for high values of the damping factor, the correction stage leads to a strong variation of the initial blank shape. At the end of the punch stroke with damping coefficients 0.8 and 1.0, the flange contour is located inside the target contour for every even iterations and for odd iterations the flange contour is located outside the target contour. These oscillations between outside and inside of the target contour are clearly controlled by the damping factor. After the first iteration, the TSE reduced depending on the damping coefficient used in the scheme. A value of 1.0 (without damping) produced maximum TSE and hence took most number of iterations to arrive at an optimal blank shape. It is evident that a value of 0.6 converges earlier than other damping coefficients. The target shape error reduces sharply to reach the target contour within few iterations as indicated in the figure. Even in the presence of over estimation of initial blank shape, the proposed algorithm is

Fig. 7 - NURBS surfaces with (a) 23, (b) 16 and (c) 11 control points. 
capable of achieving the optimal blank shape within four iterations, in the case of the studied rectangular cup deep drawing.

Hitherto, various damping coefficients were used in the blank shape optimization procedure. Since a damping coefficient of 0.6 resulted in fast convergence to optimal solution and achieves the smallest TSE within few iterations, the following sensitivity study and the optimization procedure will use 0.6 as damping coefficient.

\subsection{Influence of the number of control point}

In addition to the damping coefficients, the influence of the number of control points in the NURBS surface on the TSE is studied in the section. Three different NURBS curves with different number of control points were used to determine their influence on achieving optimal initial blank shape for the rectangular cup. The first NURBS curve was created using empirical equations and has 23 control points equally distributed over the length of the curve. This curve was then simplified by removing the less significant knots to produce second NURBS curve, using knot removal technique (Piegl and Wayne, 1997). By this technique, the less significant knots were removed and the new positions for the control points were calculated. Similarly, a third NURBS curve was produced

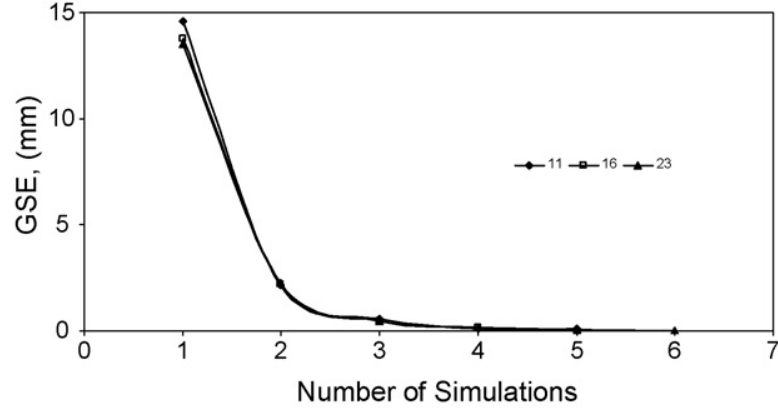

Fig. 8 - Influence of the number of control points on GSE.

by further simplifying the second NURBS curve. The second and the third NURBS curves are defined by 16 and 11 control points, respectively (Table 4). Thus, three NURBS surfaces were used separately and interpolated based on flange geometry to produce intermediate blank shapes. Fig. 7(a)-(c) shows the three initial NURBS curves and their respective control points locations. As the number of control points reduces, the shape of the NURBS surface deviates from the original surface. The deviation is more pronounced in the NURBS surface with 11 control points, Fig. 7(c).

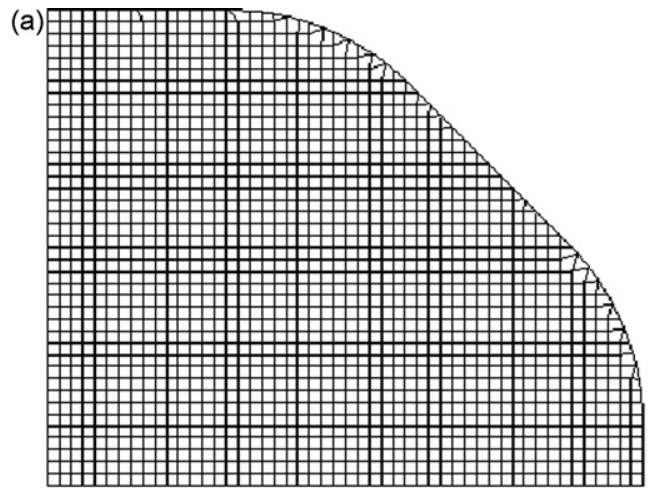

(c)

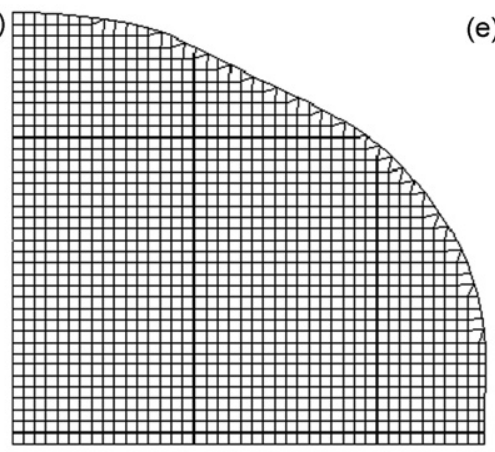

(e)

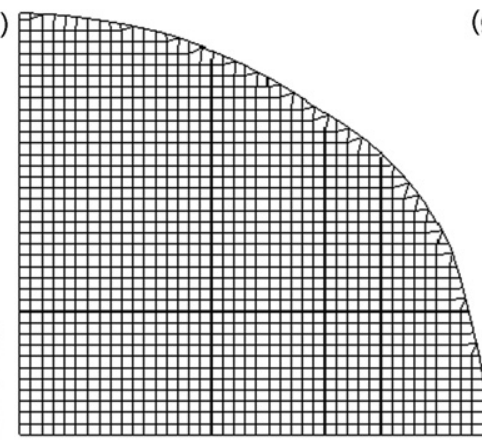

(b)

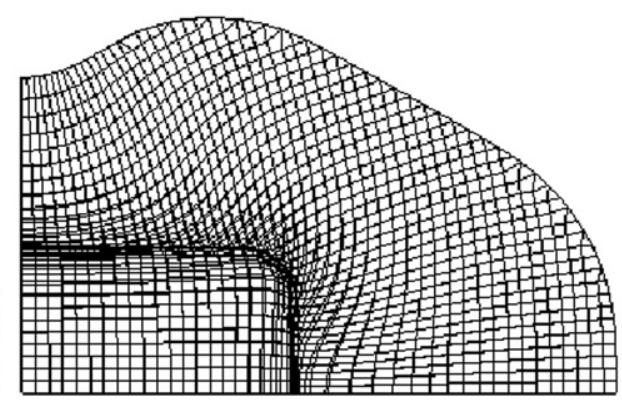

(d)

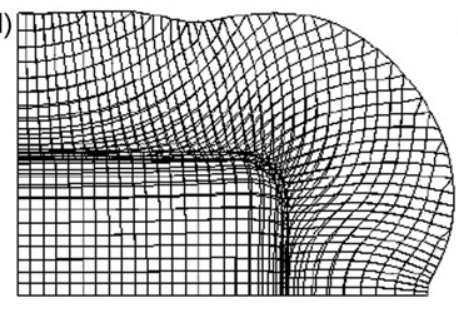

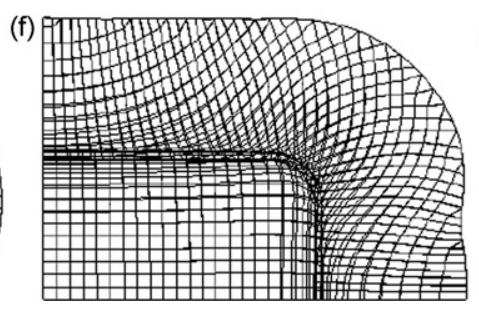

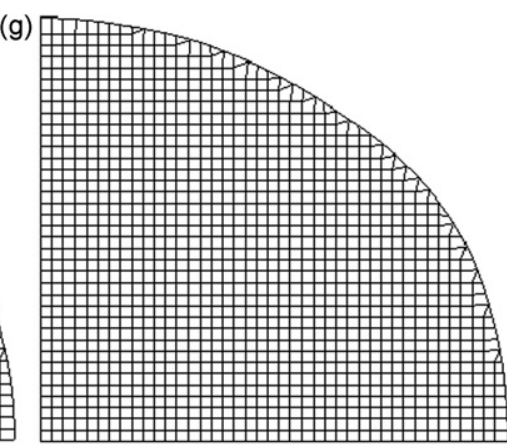

(h)

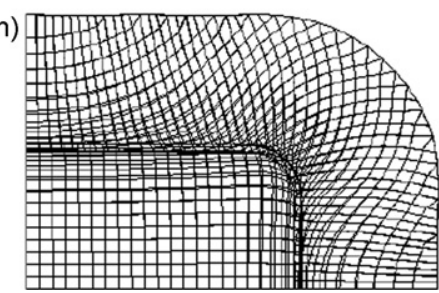

Fig. 9 - Initial blank geometries (a-d) and final contours of the deep drawn rectangular cup (e-h). 


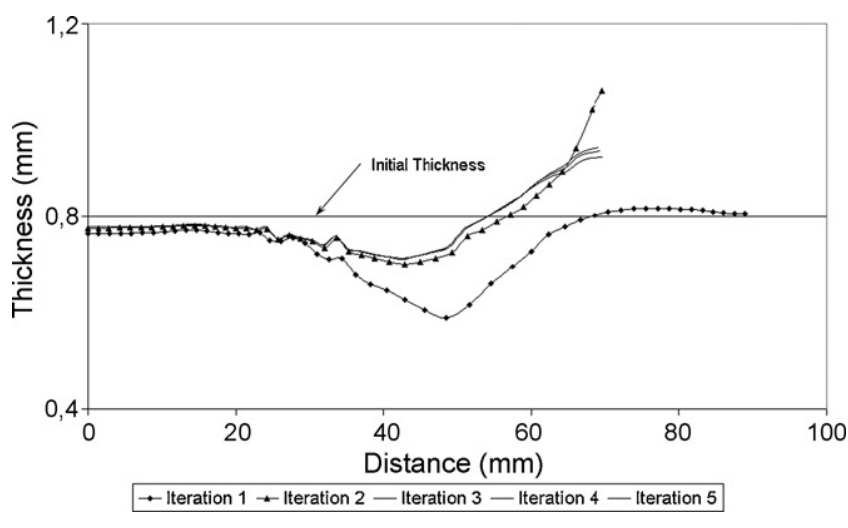

Fig. 10 - Thickness variation in rectangular cup after the iterations.

Fig. 8 shows the evolution of geometrical shape error over iterations for the three NURBS surfaces. In the first iteration, NURBS surface with 11 control points produced more error than surfaces with 16 and 23 control points. This error is a consequence of pronounced deviation of the NURBS surface with 11 control points from the original shape obtained from empirical formulae. Thereafter, the difference vanished indicating their insignificance in geometrical shape error estimation. Hence, the number of control points in the NURBS surface has little influence for the chosen mesh size. A refined NURBS surface with 23 control points was used in the remaining part of this study since it allows the evaluation of the optimal blank shape without any influence in the computation time.

\subsection{Optimal initial blank shape}

The preliminary study on the influences of the number of control points in the NURBS surface and the damping coefficient indicate that a higher number of control points and a damping coefficient of 0.6 produced best result. The empirical procedure used to obtain the initial blank, Fig. 9(a), is based on area conservancy and assumes that the thickness in the part remains the same after deep drawing. In actual practice, the blank is subjected to stretching and consequently to a thickness reduction. The result is an excess of material along the flange after deep drawing, Fig. 9(b). The proposed algorithm is then used to evaluate the differences between the flange periphery and the target contour, and the second NURBS surface is constructed. Fig. 9(c, e and g) shows the intermediate blank shapes used in deep drawing simulations. Fig. 9(d, f and h) shows the deformed cup after each iteration.

As the number of iterations increase, the flange contour gets closer to the target contour. The shape error reaches a value of $0.16 \mathrm{~mm}$ within four iterations as shown in Fig. 6. Any further iteration has a negligible impact on the required flange contour.

Fig. 10 shows the thickness profile along OX direction in the rectangular cup at the end of each iteration of the blank shape optimization procedure. In the first iteration, the blank size was large resulting in a large flange and hence the draw-in was comparatively less. Due to less draw-in, maximum thin-

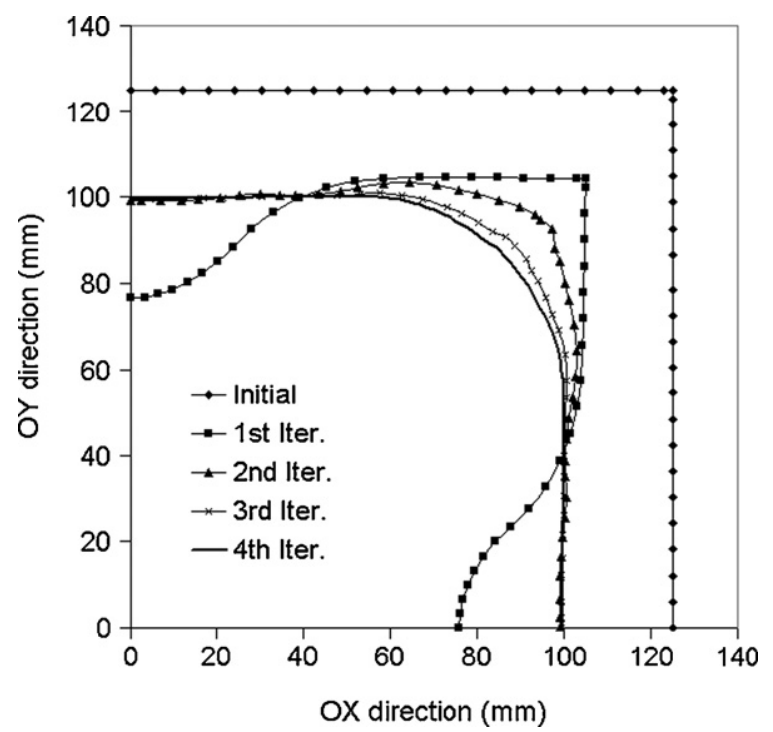

Fig. 11 - Evolution of flange contour for cross tool example.

ning occurred, especially at the punch radius. In the second iteration, a much smaller blank was utilised and consequently the thickness variation smoothed out due to a better draw-in. Thereafter, the thickness variation remained similar indicating that the chosen process parameters are optimal for the deep drawing process and results in uniform draw-in and
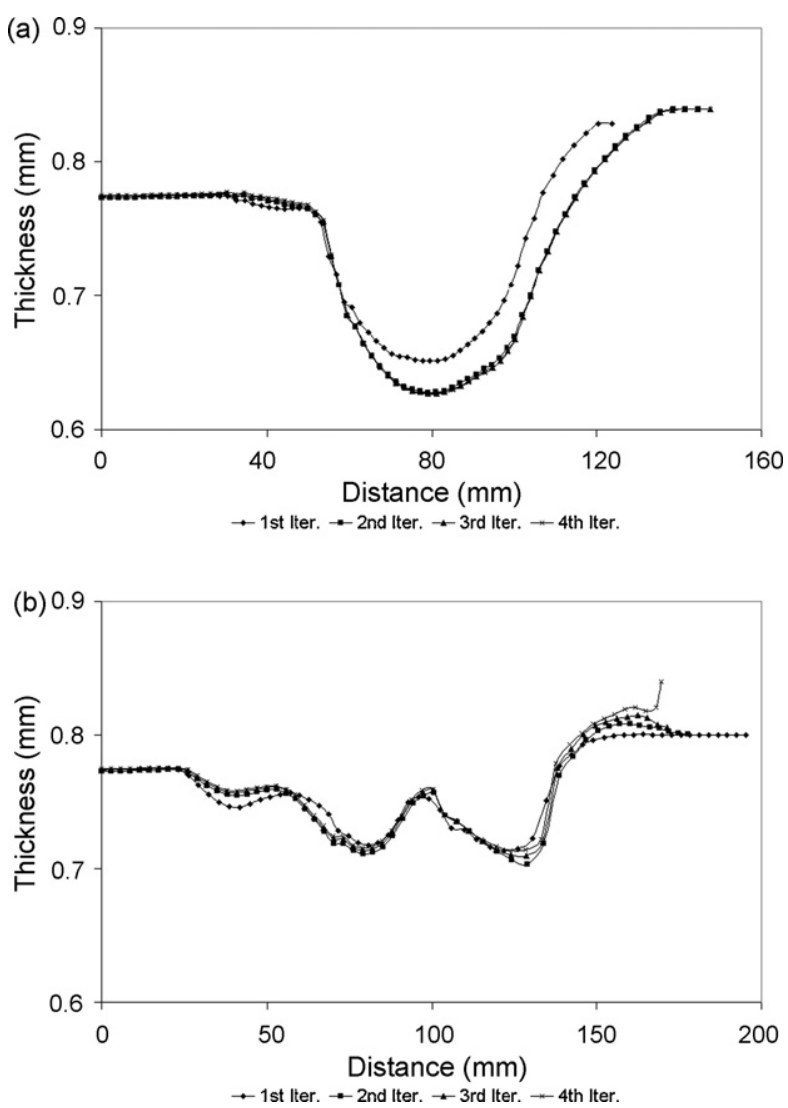

Fig. 12 - (a) Thickness variation after iterations in cross tool along ox direction. (b) Thickness variation after iterations in cross tool along diagonal direction. 
thickness distribution in the rectangular cup. The blank shape optimization procedure indirectly optimizes the thickness distribution.

A large base mesh was produced with an average element size of $2 \mathrm{~mm}$. This base mesh was cut with an initial NURBS surface, resembling a square, to produce the initial finite element mesh. Fig. 11 shows the flange contour evolution for cross tool example. The required target contour is achieved within four iterations as shown in the figure.

Fig. 12(a) shows the thickness variation in the cross tool geometry along OX direction. In the first iteration, the thickness reduction is less due to smaller initial geometry along this direction. Thereafter, from the second iteration, the thickness reduces due to a larger geometry along this direction. Negligible thickness difference occurred between iterations as shown in the figure. Fig. 12(b) shows the thickness variation along the diagonal direction. Marginal difference in thickness occurred between iterations, due to similar dimensions of the blanks along this direction.

\section{Conclusions}

The initial blank shape is one of the important process parameter in sheet metal forming that has a direct impact on the quality of the finished part, as well as on the final cost of the formed part. A new method has been proposed to determine the optimal blank shape for a formed part. A simple and straight-forward algorithm is used to compare the geometry of flange contour and target contour. Based on the correction made by the algorithm, the original mesh is trimmed using NURBS surfaces. Thus, the methodology includes the determination of initial blank shape, deep drawing simulations and a trimming scheme to achieve the final optimal blank shape. The correction to initial blank shape is made truly based on the discrepancy in the flange contour which is dictated by the material flow. Hence, the proposed method can be applied to determine optimal initial blank shape for any complex part. The sensitivity of the algorithm to the number of control points in the NURBS surface and the value of damping coefficient was also studied. For the chosen mesh, number of control points in the NURBS surface has little influence while the value of damping coefficient has large influence in quickly achieving optimal blank shape. The optimal blank shape for any industrial part can be designed within only a few iterations using the proposed methodology.

\section{Acknowledgement}

The authors are grateful to the Portuguese Foundation for Science and Technology (FCT) for the financial support for this work, through the Program POCI 2010.

\section{REF E REN C ES}

Baptista, A.J., Alves, J.L., Rodrigues, D.M., Menezes, L.F., 2006. Trimming of 3D solid finite element meshes using parametric surfaces: application to sheet metal forming. Finite Element Analysis and Design 42, 1053-1060.

Barata da Rocha, Ferreira Duarte, J., 1993. Technolgia da Embutidura. Associação Portuguesa das Tecnologias de Conformação Plástica (APTCP).

Dimas, E., Briassoulis, D., 1999. 3D geometric modelling based on NURBS: a review. Advances in Engineering Software 30, 741-751.

Guo, Y.Q., Batoz, J.L., Naceur, H., Bouabdallah, S., Mercier, F., Barlat, F., 2000. Recent developments on the analysis and optimum design of sheet metal forming parts using a simplified inverse approach. Computers and Structures 78, 133-148.

Hill, R., 1948. A theory of the yielding and plastic flow of anisotropic metals. Proceeding of the Royal Society, London A 193, 281-297.

Hu, S.M., Li, Y.F., Ju, T., Zhu, X., 2001. Modifying the shape of NURBS surfaces with geometric constraints. Computer-Aided Design 33, 903-912.

Kim, J.-Y., Kim, N., Huh, M.-S., 2000. Optimum blank design of an automobile sub-frame. Journal of Materials Processing Technology 101, 31-43.

Kuwabara, T., Si, W.-H., 1997. PC-based blank design system for deep drawing irregularly shaped prismatic shells with arbitrarily shaped flange. Journal of Materials Processing Technology 63, 89-94.

Maeder, G., 2005. Material forming and dimensioning problems: expectations from the car industry. In: Banabic, D. (Ed.), Proceedings of 8th ESAFORM conference. The Publishing House of the Romanian Academy, pp. 11-20.

Menezes, L.F., Teodosiu, C., 2000. Three-dimensional numerical simulation of the deep-drawing process using solid finite elements. Journal of Materials Processing Technology 97 (1-3), 100-106.

Oliveira, M.C., Alves, J.L., Chaparro, B.M., Menezes, L.F., 2007. Study on the influence of work-hardening modeling in springback prediction. International Journal of Plasticity 23 , 516-543.

Park, S.H., Yoon, J.W., Yang, D.Y., Kim, Y.H., 1999. Optimum blank design in sheet metal forming by the deformation path iteration method. International Journal of Mechanical Sciences 41, 1217-1232.

Piegl, L., 1991. On NURBS: a survey. IEEE Computer Graphics \& Application 11 (1), 55-71.

Piegl, L., Wayne, Tiller, 1997. The NURBS Book, second ed. Springer-Verlag, Berlin.

Sánchez, H., Moreno, A., Oyarzun, D., García-Alonso, 2003. Evaluation of NURBS surfaces: an overview based on runtime efficiency. Journal of WSCG 11. (1) (ISSN 1213-6972 WSCGǐ2003. Plzen, Czech Republic).

Tsai, M.C., Cheng, C.W., Cheng, M.Y., 2003. A real-time NURBS surface interpolator for precision three-axis CNC machining. International Journal of Machine Tools and Manufacture 43, 1217-1227. 